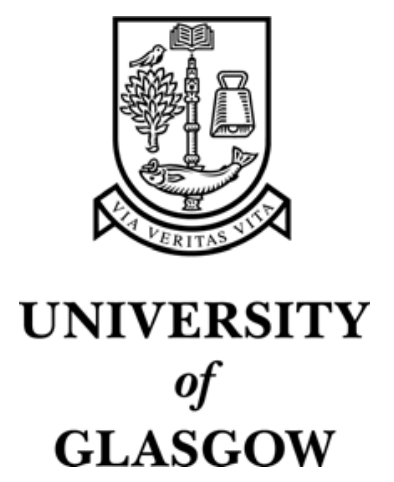

Bani-Yassein, M. and Ould-Khaoua, M. and Mackenzie, L.M. and Papanastasiou, S. and Jamal, A. (2006) Improving route discovery in ondemand routing protocols using local topology information in MANETs. In, Proceedings of the ACM International Workshop on Performance Monitoring, Measurement, and Evaluation of Heterogenous Wireless and Wired Networks, 2 October 2006, pages pp. 95-99, Torremolinos, Malaga, Spain.

http://eprints.gla.ac.uk/3544/ 


\title{
Improving Route Discovery in On-Demand Routing Protocols Using Local Topology Information in MANETs
}

\author{
M. Bani Yassein, M. Ould Khaoua, L. M. Mackenzie, S. Papanastasiou, A. Jamal \\ Department of Computing Science \\ University of Glasgow \\ Glasgow, G12 8RZ, U.K. \\ \{muneer, mohamed, lewis, stelios, jamal\}@dcs.gla.ac.uk
}

\begin{abstract}
Most existing routing protocols proposed for MANETs use flooding as a broadcast technique for the propagation of network control packets; a particular example of this is the dissemination of route requests (RREQs), which facilitate route discovery. In flooding, each mobile node rebroadcasts received packets, which, in this manner, are propagated network-wide with considerable overhead. This paper improves on the performance of existing routing protocols by reducing the communication overhead incurred during the route discovery process by implementing a new broadcast algorithm called the adjusted probabilistic flooding on the AdHoc on Demand Distance Vector (AODV) protocol. AODV [3] is a well-known and widely studied algorithm which has been shown over the past few years to maintain an overall lower routing overhead compared to traditional proactive schemes, even though it uses flooding to propagate RREQs. Our results, as presented in this paper, reveal that equipping AODV with fixed and adjusted probabilistic flooding, instead, helps reduce the overhead of the route discovery process whilst maintaining comparable performance levels in terms of saved rebroadcasts and reachability as achieved by conventional AODV. Moreover, the results indicate that the adjusted probabilistic technique results in better performance compared to the fixed one for both of these metrics.
\end{abstract}

\section{Categories and Subject Descriptors}

C.2.1 [Computer-Communication Networks]: Network Architecture and Design-Network communications

\section{General Terms}

Algorithms, Measurement, Performance, Experimentation

\section{Keywords}

MANETs, AODV, Probabilistic broadcasting. Reachability

Permission to make digital or hard copies of all or part of this work for personal or classroom use is granted without fee provided that copies are not made or distributed for profit or commercial advantage and that copies bear this notice and the full citation on the first page. To copy otherwise, to republish, to post on servers or to redistribute to lists, requires prior specific permission and/or a fee.

$\mathrm{PM}_{2} \mathrm{HW}_{2} \mathrm{~N}^{\prime} 06$, October 2, 2006, Torremolinos, Malaga, Spain.

Copyright 2006 ACM 1-59593-502-9/06/0010 ...\$5.00.

\section{INTRODUCTION}

The distributed, wireless and self-configuring nature of Mobile Ad Hoc Networks (MANETs) make them suitable for a wide variety of applications, including military operations, rescue and law enforcement missions as well as disaster recovery scenarios $[5,6]$. In AODV, whenever a source $\mathrm{S}$ needs to communicate with a destination $\mathrm{D}$, it checks for an existing route to $\mathrm{D}$ in the routing table. If the route is not present, it initiates a route discovery by broadcasting a RREQ (Route Request) packet which is flooded [1] into the network in a controlled manner, until it reaches the destination or until it reaches a node, which knows a route to the destination. Then, the destination or an intermediate node sends back a Route Reply (RREP) message, which includes the number of hops in between. Each node receiving the RREP message records a forward route to the destination and, thus, knows only the next hop required for a given route. A probabilistic approach to flooding has been suggested in $[9,1,10]$ as a means of reducing redundant rebroadcasts and alleviating the broadcast storm problem. In the probabilistic scheme, when receiving a broadcast message for the first time, a node rebroadcasts the message with a pre-determined probability $p$, thus, every node has the same probability to rebroadcast the message. When the probability is $100 \%$, this scheme reduces to simple flooding. Studies [8] have shown that probabilistic broadcasts incur significantly lower overhead compared to blind flooding while maintaining a high degree of propagation for the broadcast messages. This paper focuses on evaluating the performance of our adjusted probabilistic flooding scheme by comparing it with the flooding technique of AODV as well as a fixed probabilistic approach. The relative merits or characteristics of each method are discussed in turn and subsequent observations offer insight on possible applications of each.

To the best of our knowledge, in most previous research $[1,3,4]$ the forwarding probabilities have been set in the context of pure flooding where the goal of the broadcast algorithm has been that a packet reach every node in the network. However, in routing algorithms such as AODV the use of a broadcast is to discover a particular destination node. As a consequence, a RREQ packet does not need to reach all the nodes in the network once a particular path has been discovered that leads to the desired destination. In this paper, we implement forwarding probabilities in a dynamic and fixed manner for on-demand route discovery process in a well-known on-demand routing protocol, namely AODV. 
We have selected this routing algorithm in our present study because it is one of the early routing protocols proposed in the literature and has been widely investigated and analysed [8]. Our results reveal that equipping AODV with fixed and adjusted probabilistic flooding helps reduce the overhead of the route discovery process while maintaining a comparable performance in terms of reachability, saved rebroadcasts as achieved by conventional AODV.

The rest of this paper is organized as follows. In Section 2 , we overview related work with regards to broadcasting in MANETs. In Section 3, we describe our dynamic probabilistic approach, highlighting the difference in our approach from other similar approaches. In Section 4, we evaluate our approach and present the simulation results. Section 5 concludes the paper and offers suggestions for future work.

\section{RELATED WORK}

One of the earliest broadcast mechanisms reported in the literature is (blind) flooding, where every node in the network retransmits a message to its neighbours upon receiving it for the first time. Although flooding is very simple and easy to implement, it can be very costly and may lead to a serious problem, often known as the broadcast storm problem [8] that is characterized by high redundant packet retransmissions, network contention and collision. $\mathrm{Ni}$ et. al. [7] have studied the flooding protocol analytically and experimentally. Their obtained results have indicated that rebroadcasts could provide at best $61 \%$ additional coverage and, in the average case, only $41 \%$ over that already covered by previous transmissions. Therefore, the authors conclude that rebroadcasts are very costly and should be used with caution.

In [8], Williams et al. have classified the broadcasting techniques into the following four categories; simple flooding, probability-based, area-based, and neighbour knowledge schemes. In the flooding scheme, every node retransmits to its neighbours as a response to every newly received packet. The probability-based scheme is a simple way of controlling message floods, where each node rebroadcasts with a predefined probability $p[9]$. Obviously when $p=1$ this scheme resembles simple (blind) flooding. In the area based scheme, a node determines whether to rebroadcast a packet or not by calculating and using its additional coverage area. Of these, of interest in this study is the probabilistic scheme family of variants. In this category of broadcasting techniques, a mobile node rebroadcasts packets according to a certain probability.

Cartigny and Simplot [1] have described a probabilistic scheme where the probability $p$ of a node retransmitting a message is computed from the local density $n$ (i.e. the number of neighbours) and a fixed value $k$ for the efficiency parameter to achieve the reachability of the broadcast. This model [1] has the disadvantage of being locally uniform. Indeed, each node of a given area receives a broadcast and determines the probability according to a constant efficiency parameter (to achieve some reachability) and from the local density [1].

Zhang and Dharma [10] have also described a dynamic probabilistic scheme, which uses a combination of probabilistic and counter-based approaches. The value of a packet counter does not necessarily correspond to the exact number of neighbors from the current host, since some of its neighbours may have suppressed their rebroadcasts according to their local rebroadcast probability. On the other hand, the decision to rebroadcast is made after a random delay, which increases latency.

Bani Yassein et al. [4, 3, 2] have each proposed dynamic probabilistic broadcast schemes where the forwarding probability $p$ is dynamically adjusted by the local topology information. Topology information is obtained by proactive exchange of "HELLO" packets between neighbours.

\section{PROBABILISTIC FLOODING}

The probabilistic scheme [9] is one of the alternative approaches to simple flooding that aims to reduce redundancy through rebroadcast timing control in an attempt to alleviate the broadcast storm problem. In this scheme, when receiving a broadcast message for the first time, a node rebroadcasts the message with a pre-determined probability $p$ so that every node has the same probability to rebroadcast the message, regardless of its number of neighbours.

In dense networks, multiple nodes share similar transmission ranges. Therefore, these probabilities control the frequency of rebroadcasts and thus might save network resources without affecting delivery ratios. Note that in sparse networks there is much less shared coverage; thus some nodes will not receive all the broadcast packets unless the probability parameter is high. So if the rebroadcast probability $p$ is set to a far smaller value, reachability will be poor. On the other hand, if $p$ is set to a far larger value, many redundant rebroadcasts will be generated. The need for dynamic adjustment, thus, rises.

The rebroadcast probability should be set high at the hosts in sparser areas and low at the hosts in denser areas. Our simple method for density estimation requires mobile hosts to periodically exchange "HELLO" messages between neighbours to construct a 1-hop neighbour list at each host. A high number of neighbours implies that the host is in a dense area, whilst a low number of neighbors implies that the host is situated in a sparser area. We increase the rebroadcast probability if the value of the number of neighbours is too low (or similarly if the current node is located in a sparse neighbourhood), which indirectly causes the probability at neighbouring hosts to be incremented. Similarly, we decrease the rebroadcast probabilities if the value of number of neighbours is too high.

This kind of adaptation causes a dynamic stability between rebroadcast probabilities and the number of neighbours among neighbouring hosts. Intuitively, the probabilities at the stability states should lead to optimal solutions. We adopt a simple adaptation algorithm. A brief outline of the adjusted probabilistic flooding algorithm is presented in Algorithm 1 and operates as follows. On hearing a broadcast message $m$ at node $X$, the node rebroadcasts a message according to a high probability if the message is received for the first time, and the number of neighbours of node $X$ is less than average number of neighbours typical of its surrounding environment. Hence, if node $X$ has a low degree (in terms of the number of neighbours), retransmission should be likely. Otherwise, if $X$ has a high degree its rebroadcast probability is set low.

The adjusted rebroadcast probability for probabilistic broadcasting algorithm for each node is briefly presented in Algorithm 1 and fully described in [4].

Our algorithm is a combination of the probabilistic and knowledge based approaches. It dynamically adjusts the re- 


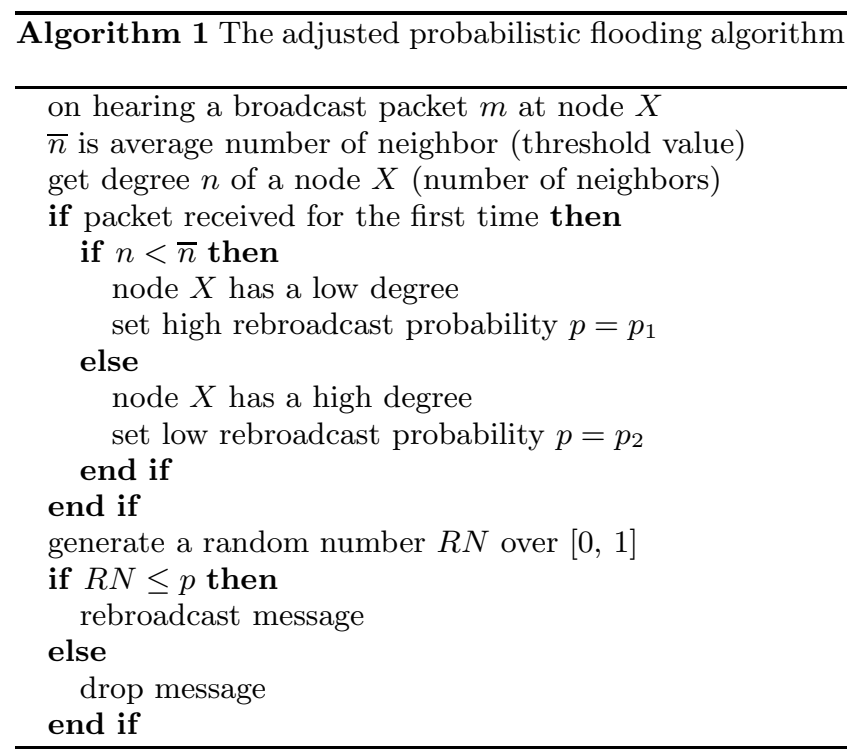

broadcast probability $p$ at each mobile host according to the value of the local number of neighbours. The value of $p$ changes when the host moves to a different neighbourhood. In a sparser area, the rebroadcast probability is larger and in denser area, the probability is lower. Compared with the probabilistic approach where $p$ is fixed, our algorithm achieves higher saved rebroadcast. Also, the decision to rebroadcast is made immediately after receiving a packet in our algorithm without any delay.

We present an estimate of the average number of neighbours as the basis for the selection of the value of $p$. Let $A$ be the area of an ad hoc network, $N$ be the number of mobile hosts in the network. The average number of neighbour can be obtained as shown below.

$$
\bar{n}=(N-1) * 0.8 * \frac{\pi^{2}}{A}
$$

\section{PERFORMANCE EVALUATION}

In this section, we evaluate the performance of the adjusted probability probabilistic algorithm against the fixed rebroadcast probability and flooding. We have implemented all three algorithms in the AODV protocol. The metrics for comparison include the saved rebroadcasts and reachability.

We have used the ns-2 simulator (v.2.27) to conduct extensive experiments to evaluate the performance of probabilistic flooding. The original AODV protocol uses simple flooding to broadcast routing requests. We have implemented two AODV variations: one using probabilistic method with Fixed Probability, and the other using our adjusted probabilistic algorithm. The main idea of both is to reduce the no. of rebroadcasts during route discovery, thereby reducing the network traffic and decrease the probability of channel contention and packet collision.

Since our algorithm is based on a probabilistic approach it does not fit every scenario, as there is a small chance that the route requests cannot reach the destination. It is necessary to re-generate the route request if the previous route request failed to reach the destination. The AODV protocol, on the contrary, uses flooding in the route discovery phase, which means that all route requests will reach their destinations if

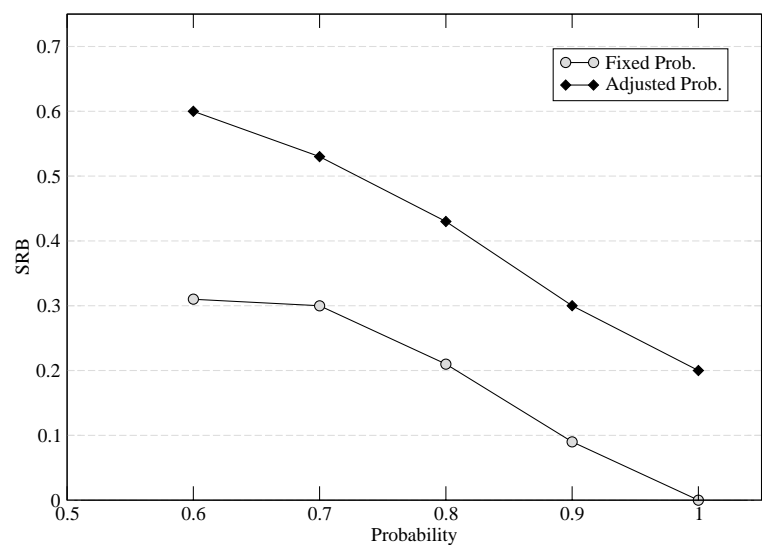

Figure 1: SRB vs rebroadcast probability with node speed $10 \mathrm{~m} / \mathrm{s}$

Table 1: Parameters used in the simulation

\begin{tabular}{|c|c|}
\hline Parameter & Value \\
\hline Transmitter range & 250 meters \\
\hline Bandwidth & $2 \mathrm{Mbps}$ \\
\hline IF queue length & 50 frames \\
\hline Simulation time & 900 secs \\
\hline Pause time & 0 secs \\
\hline Packet size & 512 bytes \\
\hline Topology size & $600 \times 600 \mathrm{~m}$ \\
\hline Number of nodes & $25,50,75,100$ \\
\hline Maximum speed & $10 \mathrm{~m} / \mathrm{sec}$ \\
\hline
\end{tabular}

the network is not partitioned. Based on this observation, our algorithm should perform better than AODV in dense networks.

The network considered for the performance analysis of the rebroadcast probability vs network density varies from 25 to 100 nodes placed randomly on $600 \times 600 \mathrm{~m}$ area, with each node engaging in communication transmitting within 250 meter radius and having bandwidth of $2 \mathrm{Mbps}$. The random waypoint model is used to simulate 25 mobility patterns with retransmission probabilities ranging from 0.5 to 1.0 percent with 0.1 percent increment per trial.

In short, the random waypoint model considers nodes that follow a motion-pause recurring mobility state. Each node at the beginning of the simulation remains stationary for some pause time seconds, then chooses a random destination and starts moving towards it with speed selected from a uniform distribution (0, max_speed]. After the node reaches that destination, it again stands still for a pause time interval and picks up a new destination and speed. This cycle repeats until the simulation terminates. The maximum speed of $10 \mathrm{~m} / \mathrm{sec}$ and pause times of 0 seconds are considered for the purposes of this study. The simulation parameters are summarised in Table 1.

The performance of broadcast protocols can be measured by a variety of metrics $[5,8,7]$. A commonly used metric is the number of message re-transmissions with respect to the number of nodes in the network [8]. In this work, we use rebroadcast savings, which is a complementary measure and is precisely defined below. The next important metric is reachability, which is defined in terms of the ratio of nodes 


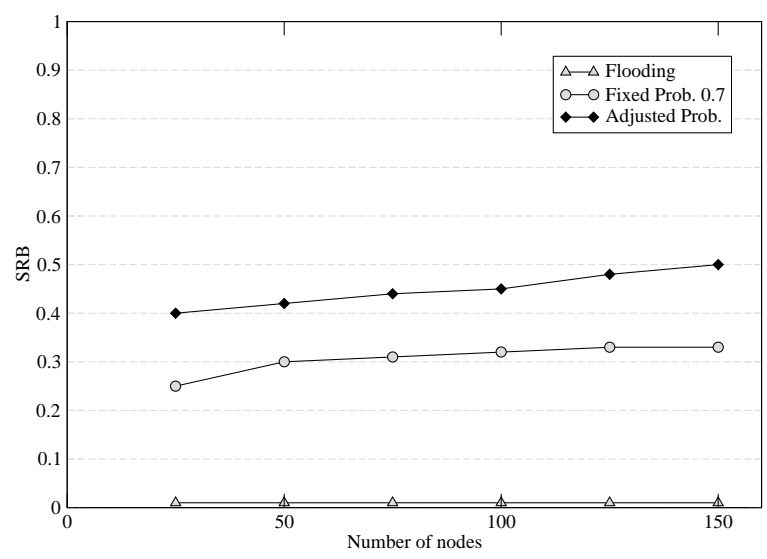

Figure 2: SRB of three broadcast schemes vs network density with node speed of $10 \mathrm{~m} / \mathrm{s}$

that received the broadcast message out of all the nodes in the network. The formal definitions of these two metrics are given as follows [7].

Saved ReBroadcasts (SRB) : Let $r$ be the number of nodes that received the broadcast message and let and $t$ be the number of nodes that actually transmitted the message. Saved rebroadcast is then defined by $(r t) / r$.

Reachability (RE) : is the percentage of nodes that received the broadcast message to the total number of nodes in the network. For meaningful information, the total number of nodes should include those nodes that are part of a connected component in the network. For disconnected networks this measure should be applied to each of the components separately.

We have compared the saved broadcast (SRB) in fixed probability and our adjusted probabilistic algorithm. Figure 1 shows that our algorithm can significantly reduce SRB with rebroadcast probabilities ranging from 0.5 to 1.0 percent with 0.1 percent increment per trial for a network of 50 nodes and maximum speed $20 \mathrm{~m} / \mathrm{s}$ and 0 pause time. Figure 2 shows the SRB of the fixed probabilistic scheme against our adjusted probabilistic algorithm.

The SRB of adjusted probabilistic is $40 \%$ in low-density networks (25 nodes) and 50\% in high-density networks (150 nodes). The SRB of the fixed probabilistic scheme with the probability assigned to 0.7 in any density of network is around $30 \%$. Figure 3 shows that reachability increases when network density increases, regardless of what kind of the algorithm is used. The simple flooding method has the best performance in reachability, as expected. The performance of adjusted probabilistic algorithm shows that the reachability is above $95 \%$ in any density of the network. In all network densities, the reachability of our algorithm performs better than the probabilistic scheme when probability Is set to 0.7. In higher density networks, i.e. for 120 hosts and above, the reachability of our approach and flooding are evenly matched, with both performing very adequately (close to $100 \%$ ). We have noted that the extra redundancy of RREQ transmissions is what results in more contention and collisions. Considering all the previous results, the adjusted probabilistic-enabled AODV is shown to

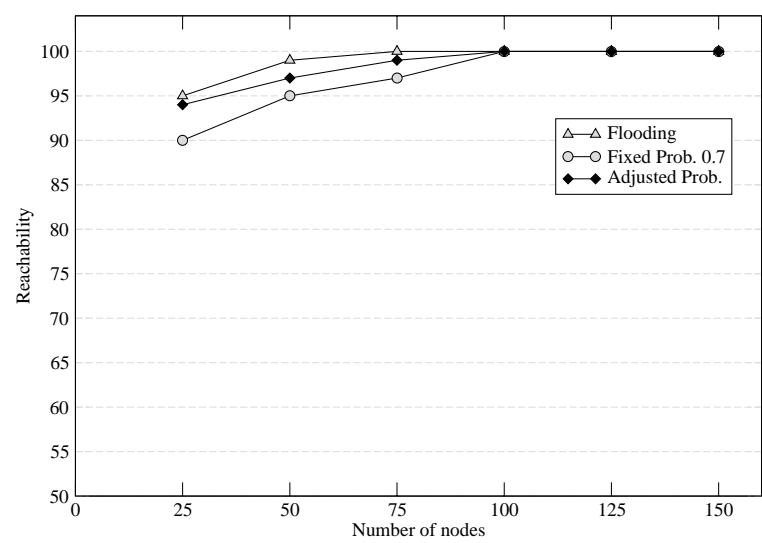

Figure 3: Reachability of three broadcast algorithms

improve AODV performance in all aspects for scenarios with low mobility.

\section{CONCLUSIONS}

This paper has weighed up the performance of adjusted probabilistic flooding on the AODV protocol, which traditionally uses simple flooding, in order to increase saved rebroadcasts of route requests. This proposed algorithm determines the rebroadcast probability by taking into account the network density. In order to improve the saved rebroadcasts, the rebroadcast probability of low density nodes is increased while that of high density nodes is decreased. Compared with simple flooding, our simulation results have shown that the adjusted probabilistic flooding algorithm can improve the saved broadcast up to $50 \%$ without affecting reachability, even under conditions of high mobility and density.

As a prospect for future work, we plan to evaluate the performance of adjusted probabilistic flooding on the Dynamic Source Routing (DSR) algorithm. Then we aim to build an analytic model for our approach in order to facilitate the exploration of the optimal adaptation strategy, with regard to probability setting and network density. Finally, since the technique avails itself to various types of network-wide dissemination, we plan to integrate it with a proactive routing protocol, namely OLSR. This protocol already incorporate techniques which reduce the effect of flooding and are orthogonal to our scheme, which implies the opportunity to examine if cumulative improvements with our method are possible.

\section{REFERENCES}

[1] J. Cartigny and D. Simplot. Border node retransmission based probabilistic broadcast protocols in ad-hoc networks. Telecommunication Systems, 22(1-4):189-204, 2003.

[2] L. M. M. M. Bani Yassein, M. Ould-Khaoua and S. Papanastasiou. Improving the performance of probabilistic flooding in manets. In Proceedings of International Workshop on Wireless Ad-hoc Networks (IWWAN-2005), May 2005.

[3] L. M. M. M. Bani-Yassein, M. Ould Khaoua and S. Papanastasiou. Applications of highly adjusted probabilistic broadcasting in manets. In Proceedings of 
the 4 th International Multiconference on Computer Science and Information Technology CSIT 2006, pages 469-476, April 2006.

[4] L. M. M. M. Bani-Yassein, M. Ould-Khaoua and S. Papanastasiou. Performance analysis of adjusted probabilistic broadcasting in mobile ad hoc networks. International Journal of Wireless Information Networks, pages 1-14, March 2006. Springer Netherlands.

[5] W. Peng and X.-C. Lu. On the reduction of broadcast redundancy in mobile ad hoc networks. In MobiHoc '00: Proceedings of the 1st ACM international symposium on Mobile ad hoc networking $\&$ computing, pages 129-130, Piscataway, NJ, USA, 2000. IEEE Press.

[6] C.-K. Toh. Ad Hoc Mobile Wireless Networks: Protocols and Systems. Prentice-Hall, 2002.
[7] Y.-C. Tseng, S.-Y. Ni, Y.-S. Chen, and J.-P. Sheu. The broadcast storm problem in a mobile ad hoc network. Wireless Networks, 8(2/3):153-167, 2002.

[8] B. Williams and T. Camp. Comparison of broadcasting techniques for mobile ad hoc networks. In MobiHoc '02: Proceedings of the 3rd ACM international symposium on Mobile ad hoc networking 86 computing, pages 194-205, New York, NY, USA, 2002. ACM Press.

[9] D. C. Y. Sasson and A. Schiper. Probabilistic broadcast for flooding in wireless mobile ad hoc networks. Technical Report IC/2002/54, EPFL, 2002.

[10] Q. Zhang and D. Agrawal. Dynamic probabilistic broadcasting in manets. Journal of Parallel Distributed Computing, 65:220-233, 2005. 